\title{
The Use of Machine Learning for Correlation Analysis of Sentiment and Weather Data
}

\author{
$\mathrm{Hu}$ Li, Zahra Jadidi, Jinyan Chen and Jun Jo \\ School of Information and Communication Technology \\ Griffith University, Southport QLD 4222, Australia \\ \{hu.li, z.jadidi, jinyan.chen and j.jo\}@griffith.edu.au
}

\begin{abstract}
The development of the Internet of Things (IoT) drives us to confront, manage and analyse massive and complicated data generated from various sensors. Also, social media have rapidly become very popular and can be considered as important source of data. Twitter on average generates about 6,000 tweets every second, which total to over 500 million tweets per day. Facebook has over 2 billion monthly active users. The individual posts may be trivial, however, the accumulated big data can provide diverse valuable information, which can be also correlated with IoT and enable human sentiment identification of the environment changes. This work proposes a machine learning model for correlation analysis and prediction of weather conditions and social media posts. In experimental evaluation we demonstrate that the Big Data analysis approach and machine learning techniques can be used to analyse and predict sentiment of different weather conditions.
\end{abstract}

\section{Introduction}

With the rapid growth of the Internet and smartphone users, social media play important roles in our daily lives. Millions of 'connected' citizens share information through social media and the enormous amount of posts often turns into collective knowledge or valuable resources for the analysis and management of our environment. It is now recognised that such non-traditional approaches present inexpensive means for gathering rich, authentic, and unsolicited data on people's perceptions and experiences [1].

Big data is described as datasets that are diverse, volumes, complex and is not easy to handle by traditional database technologies. Compared with traditional datasets, big data typically includes masses of unstructured data, such as social media posts, structured data, data collected from various sensors, therefore it provides great potential for real-time analysis. Cloud computing usually provides big data with infrastructure of cost efficiency, elasticity, and easy management.

In the paradigm of IoT, we investigate how the public opinions from social media are related to the weather data in the context of human sentiments. This work builds on top of work related to sentiment analysis and environmental changes [3], [4]. Findings of this work will expand the extent of the IoT technology to predict human sentiments for different weather conditions. 
This work relied on Twitter data posted from Cairns city in Queensland, Australia. In line with previous research this work was conducted on Hadoop cluster, running MongoDB and NoSQL databases where Twitter posts have been stored, and relational MySQL database to store IoT data. Data and processing were done in the distributed environment across nodes in a shared-nothing architecture. Utilization of artificial neural network has been proposed. A two-layer feed-forward neural network (with one hidden layer) was employed to predict sentiment pattern using non-linear regression [13]. A fitnet method was used in Matlab to simulate this fitting neural network [12]. A machine learning model was trained for the Twitter dataset and the accuracy of the prediction was tested.

\section{Big Data}

The amount of digital data is growing at an exponential rate producing data commonly referred as Big Data. Big Data is a term for datasets that are so large or complex that the traditional data processing methods are inadequate to deal with them. Big data challenges include issues related to capturing, storing, analysis, sharing, visualization, as well as information privacy [5]. Lately, the term "big data" tends to refer to the use of predictive analytics, user behavior analytics, and other advanced data analytics methods that extract value from data [6]. Defined challenges and opportunities brought about by increased data with a $3 \mathrm{Vs}$ model, i.e., the increase of Volume, Velocity, and Variety [14]. In the "3Vs" model, Volume means a massive amount of data; Velocity means the data collection and that the analysis must be rapidly and timely conducted; Variety indicates the various formats and types of data. A huge amount of valuable data can be generated by crawling the Internet, from forum posts, chatting records, and microblog messages. Such Internet data may be valueless individually, but through the analysis of accumulated data and their correlation, useful information can be identified. It is even possible to forecast users' behaviours and emotional moods. Traditional data management and analysis systems are based on the relational database management system (RDBMS). However, such RDBMSs only apply to structured data, other than semi-structured or unstructured data. The traditional RDBMSs could not handle the huge volume and heterogeneity of big data [14]. Such data sources include sensors, videos, clickstreams, and other data sources. In the paradigm of IoT, sensors all over the world are collecting and transmitting data to be stored and processed in the cloud [5].

\section{Sentiment Analysis on Social Media Data}

Sentiment analysis is a new approach to text analysis, which aims at determining the opinion and subjectivity of reviewers. Sentiment relates to valence and reflects underlying emotions, broadly classified into positive, neutral and negative. Sentiment can be extracted from social media statements through the use of computational linguistics and natural language processing [2]. Specifically, in this work we relied on Twitter as a source of, because it is a relatively commonly used platform and some of the 
Twitter data through public API is freely available for analysts [7]. Twitter on average has about 6,000 tweets every second, which total to over 500 million tweets per day. These posts can provide valuable information of diverse topics. There are many sentiment analysis methods for English text presented in literature. The existing work on sentiment analysis can be classified into three popular groups based on methods employed [8]. Machine learning method, lexicon-based approach and rule-based approach.

Another classification is oriented more on the structure of the text: document level, sentence level or word/feature level classification. In general sentiment analysis of tweets has been coded into positive, negative or neutral [9]. To obtain higher accuracy it has been proposed to use classifiers that are trained with large volume of human annotated text [10]. Further improvements can be obtained over the time via deep (machine) learning. Accuracy of the computer-based classifier depends, and it has been reported from $41.9 \%$, for a sample of Thai students based on tweets about Bangkok and Phuket, to $66 \%$ related to hotel reviews [11].

Considering that in this work we analyse sentiment of social media post we utilized Valence Aware Dictionary for Sentiment Reasoning (VADER) approach for sentiment analysis because it is purposely developed for sentiment analysis calculation of short text found in social media posts [6].

\section{The Sentimental Analysis with Weather Data}

For this research, the collected Twitter data is stored in a NoSQL MongoDB database, which is located on a cluster computer with a Hadoop architecture. Each Twitter data in the database contains Metadata, including the content of the tweet, language, location where account was opened, and potentially place from which the Twitter message was sent, if GPS is enabled. We found that on average $15 \%$ of tweets have their GPS enabled.

Twitter users send tweets for a wide range of reasons and some of tweets refer to the topic. Therefore, it needs to be filtered to extract messages of interest. All keywords were extracted using a case insensitive search technique, and variations of the same word (e.g. 'dive', 'diving') were compiled as the same keyword. Tweets were analysed with regards to their positive or negative polarity.

As indicated above method adopted by this research is VADER rule-based approach with the text-level, which combines a general lexicon and a series of intensifiers, punctuation transformation, emoticons, and many other heuristics to compute sentiment polarity. The VADER sentiment lexicon is composed of more than 7000 items with associated sentiment intensity validated by humans. The sentiment score ranges from minus one (negative) to plus one (positive), with the zero being 'neutral'.

\section{Twitter Dataset}

This paper considers the impact of weather variables on users' mood during daytime. In this regard, the Twitter data are mapped with weather information in Cairns. The 
Bureau of Meteorology (http://www.bom.gov.au), which is an Australia national weather, climate, and water agency is used to find the relevant weather data. These data can also be obtained through APi provided as Open Data. The tweets mapped to the weather information are eventually labelled with hourly sentiment scores.

The Twitter dataset used in this paper consists of tweets posted between 8:00 am to 5:00 pm (October 2016 to October 2017) from Cairns in Australia. This study aims to find the correlation between weather conditions and sentiment scores. An artificial neural network (ANN) was used to predict sentiment values in new data. The ANN model was trained and evaluated with training and testing datasets respectively. $70 \%$ of the input data is used for training dataset and $30 \%$ is reserved for testing dataset. Table 1 shows the number of samples in each dataset.

Table 1. Samples in training and testing datasets

\begin{tabular}{|l|c|c|c|}
\hline Dataset & Total Samples & $\begin{array}{c}\text { Training dataset } \\
\mathbf{( 7 0 \% )}\end{array}$ & $\begin{array}{c}\text { Testing dataset } \\
\mathbf{( 3 0 \% )}\end{array}$ \\
\hline $\begin{array}{l}\text { Carins } \\
\text { (Oct2016-Oct2017) }\end{array}$ & 55216 & 38651 & 16564 \\
\hline
\end{tabular}

\section{Sentiment pattern analysis}

The Twitter dataset is very noisy and we need to remove noise to be able to analyze the sentiment patterns. A smoothing spline method is used to find the fitted curve for this noisy dataset. Figure 1 shows the relationship between temperature, humidity, wind, rainfall, and sentiment in our Twitter dataset collected from Cairns Australia.

The $\mathrm{x}$-axes in all graphs were rescaled considering the maximum value for the variables in the dataset. This helps to convert $\mathrm{x}$-axis to a dimensionless value in the [0 1] range and compare the impact of different variables on sentiment. According to the figure, the concerned tweets were in the range of $22-32^{\circ} \mathrm{C}$ (temperature), $47-87 \%$ (humidity), $10-31 \mathrm{~km} / \mathrm{h}$ (wind speed) and rain less than $14 \mathrm{~mm}$. The averaged sentiment values for this ranges are positive. It shows that these ranges were desirable conditions for users and made them feel positive. Although there are some negative sentiments in these ranges, the averaged sentiment for each weather variable is positive. 

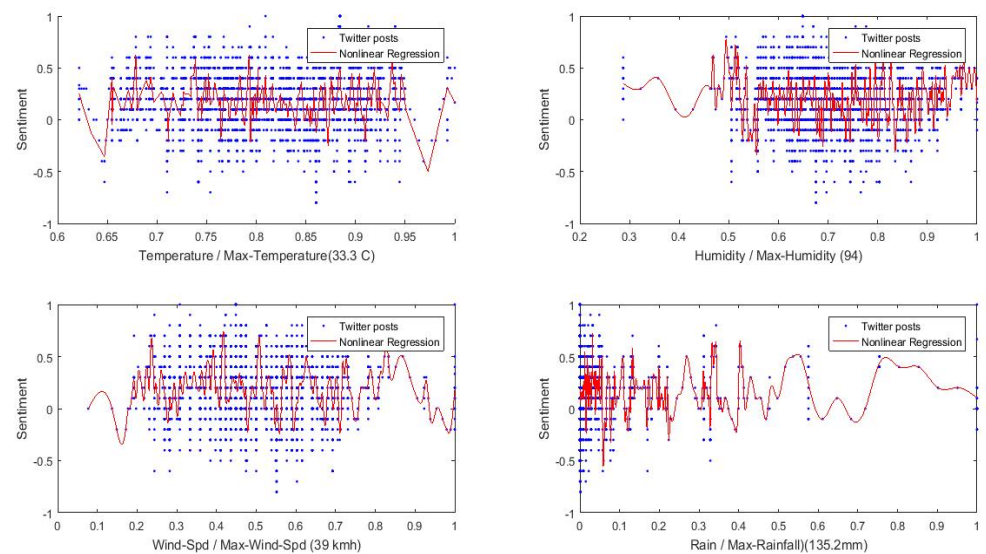

Figure 1. Comparison of the sentiments.

\section{Experimental Results}

One of the objectives of this study was to forecast human sentiments under certain weather conditions using an artificial neural network. In this regard, a two-layer feedforward neural network (with one hidden layer) is employed to predict sentiment pattern using non-linear regression [13]. A fitnet method was used in Matlab to simulate this fitting neural network [12]. The parameters of this neural network predictor (NNP) is provided in Table 2:

Table 2. Parameters of NNP

\begin{tabular}{|l|l|}
\hline Description & Values \\
\hline Number of neurons in hidden layer & 30 \\
\hline Number of input features & 8 \\
\hline Training function & Levenberg-Marquardt (Trainlm) \\
\hline Number of Ephocs & 5000 \\
\hline
\end{tabular}

The NNP has [8 30 1] topology, and it is simulated in Matlab 2016a. For measuring the performance of NNP, Accuracy is defined as Eq. (1):

$$
\operatorname{Accuracy}(\%)=\frac{C_{p}}{C_{p}+W_{p}} * 100
$$

Where $\mathrm{C}_{\mathrm{p}}$ is the number of correct predictions and $\mathrm{W}_{\mathrm{p}}$ shows the number of wrong predictions. The NNP method is trained with 5000 Ephocs. Afterwards, the trained method is separately evaluated with training dataset and testing dataset (new data). The results are as Table 3. In addition, Mean Square Error (MSE) is shown in Figure 2. All experiments are repeated 10 times to achieve higher accuracy, and all provided 
results are averaged values of 10 trials. The NNP method could predict $95 \%$ of the sentiment values in new dataset.

Table 3. Comparison of the Accuracy of FFNNP trained with different algorithms

\begin{tabular}{|l|l|l|}
\hline Feed-forward training algorithms & \multicolumn{1}{|c|}{$\begin{array}{c}\text { Accuracy with Training } \\
\text { dataset (\%) }\end{array}$} & $\begin{array}{c}\text { Accuracy with Testing dataset } \\
(\%)\end{array}$ \\
\hline Trainlm & 98 & 95 \\
\hline Traingd & 97.5 & 93.6 \\
\hline Trainbr & 96.8 & 94.8 \\
\hline
\end{tabular}

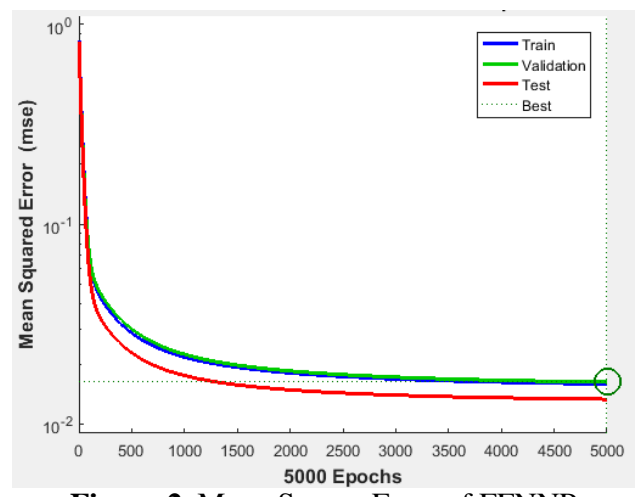

Figure 2. Mean Square Error of FFNNP

NNP was trained with trainlm and the performance was compared with other training algorithms in Table 3. As the result shows, trainlm has the highest accuracy over 10 trials (95\% correct prediction). In addition, MSE function shown in Figure 2 is used to evaluate the performance of NNP. In this phase, $70 \%$ of input data is used for training dataset and 30\% is for both validation and testing datasets. As Figure 2 shows, MSEs in all data sets are about 0.02 after 3000 epochs. This also shows the high performance of NNP in predicting sentiments in new data.

\section{Conclusion}

This paper introduced Big Data concept to analyse and predict human sentiment depending on different weather conditions. Twitter social media post are used and we utilized VADER rule based sentiment analysis method to compute sentiment polarity. Additionally, BOM Open weather data have been captured. The correlation between the two has been investigated. A machine learning model was trained for the Twitter dataset and the accuracy of the prediction was tested. The analysis revealed that the Twitter-based sentiment analysis demonstrated a close relationship between different weather conditions and users' sentiments. The results show that the Big Data analysis and machine learning techniques can be used to analyse and predict sentiment to different weather conditions. It also revealed comfortable range of weather conditions to humans. 


\section{Acknowledgement}

This project was partly funded through a National Environment Science Program (NESP) fund, within the Tropical Water Quality Hub (Project No: 2.3.2).

\section{References}

1. O'Leary, D., (2011). The use of social media in the supply chain: survey and extensions. Intelligent System Account. Finance Manag. 18 (2e3), 121e144.

2. Thelwall, M., Buckley, K., Paltoglou, G., Cai, D. and Kappas, A. (2010), Sentiment strength detection in short informal text. J. Am. Soc. Inf. Sci., 61: 2544-2558. doi:10.1002/asi.21416

3. Becken, S., Stantic, B., Chen, J., Alaei, A. R., \& Connolly, R. M. (2017). Monitoring the environment and human sentiment on the Great Barrier Reef: Assessing the potential of collective sensing. Journal of Environmental Management, 203, 87-97.

4. Chen, J., Wang, S., \& Stantic, B. (2017). Connecting Social Media Data with Observed Hybrid Data for Environment Monitoring. In International Symposium on Intelligent and Distributed Computing (pp. 125-135).

5. Chen, M., Mao, S., \& Liu, Y. (2014). Big data: A survey. Mobile Networks and Applications, 19(2), 171-209.

6. Laney D (2001) 3-d data management: controlling data volume, velocity and variety. META Group Research Note.

7. Chaffey, C., 2016. Global social media research summary, (2016). Smart Insights.

8. Collomb, A., Costea, C., Joyeux, D., Hasan, O., \& Brunie, L. (2014). A study and comparison of sentiment analysis methods for reputation evaluation. Rapport de recherche RRLIRIS-2014-002.

9. Philander, K. and Zhong, Y.Y. (2016) Twitter sentiment analysis: Capturing sentiment from integrated resort tweets. International Journal of Hospitality Management, 55, 16-24.

10. Claster, W., Pardo, P., Cooper, M. and Tajeddini, K. (2013) Tourism, travel and tweets: algorithmic text analysis methodologies in tourism. Middle Eastern Journal of Management, $1(1), 81-100$.

11. Kasper, W. and Vela, M. (2011) Sentiment Analysis for Hotel Reviews, Proceedings of the Computational Linguistics-Applications Conference, 45-52.

12. https://au.mathworks.com/help/nnet/ref/fitnet.html

13. Catalão, J. P. D. S., Mariano, S. J. P. S., Mendes, V. M. F., \& Ferreira, L. A. F. M. (2007). Short-term electricity prices forecasting in a competitive market: A neural network approach. Electric Power Systems Research, 77(10), 1297-1304.

14. Stantic B., Pokorný J., (2014), Opportunities in Big Data Management and Processing. DB \&IS, $15-26$ 